\section{Detection of Antibody Display Phage without Clearing of Bacterial Culture}

BioTechniques 29:737-740 (October 2000)

Filamentous phage display has become a powerful method for the discovery of affinity-binding reagents (6). Linear and constrained peptides, antibody fragments (scFvs and Fabs) and some alternative binding domains have all been displayed on phage particles by fusing to one of the phage coat proteins. Although several phage proteins (derived from gVIII, gVI, gVII and gIX) have all been used as fusion partners for display of recombinant proteins, gIII is most widely used for the display of antibody fragments. Phagemids that contain a phage origin of replication, an antibiotic resistance marker and the gene encoding the antibody/gIII fusion protein are readily constructed with conventional molecular biology techniques. Through largescale ligation, transformation and recombination strategies, large libraries of $10^{8}-10^{11}$ different recombinants are now being generated for use in affinity selection strategies $(1,4,5)$.

Once a library of potential binding agents for phage display is generated, individual phage with the capacity to bind to a chosen protein target must be isolated from an enormous excess of non-binding phage. Typically, a target antigen is immobilized on a solid support and incubated with an aliquot of the phage library. Phage particles that remain attached to the antigen after removal of the unbound fraction can be eluted by $\mathrm{pH}$ denaturation or enzymatic cleavage and amplified by reinfection of exponentially growing E. coli. Phage that are generated following this amplification are used as the input in the subsequent cycle of affinity selection (panning). After several rounds of panning, E. coli are reinfected with the enriched phage, and individual clones are isolated. A helper phage (e.g., M13KO7) is used to rescue the phagemid into an infectious phage particle displaying the selected binding domain. These individual phage stocks are screened with ELISA to find the clones with the desired binding properties. Traditionally, overnight cultures of phage-producing bacteria are centrifuged to pellet the bacteria, and the phage supernatants are used in the ELISA (2). Alternatively, phage can be purified and concentrated from cleared supernatants by polyethylene glycol precipitation. Although performing this procedure on a small scale may seem reasonable, developing high-throughput screening procedures requires the investigator to eliminate costly, timeconsuming or unnecessary steps. In our laboratory, centrifugation of cultures limited the throughput in screening isolated clones using phage ELISA.
We have generated phage-displaying scFvs or Fabs by PCR amplification of cDNA corresponding to the heavy and light chain variable regions from the HP6002, HP6025 and HP6054 hybridomas (3); cells, obtained from ATCC (Manassas, VA, USA) included CRL-1788, CRL-1775 and CRL-1763, respectively. Assembled scFvs or Fabs were digested with SfiI and NotI, subcloned into the pCANTAB5E vector (Amersham Pharmacia Biotech, Piscataway, NJ, USA) and transformed into TG1 or XL1-Blue-competent E. coli. Individual clones capable of specific binding to the target antigen were isolated by conventional methods and then used to explore alternatives to centrifu-

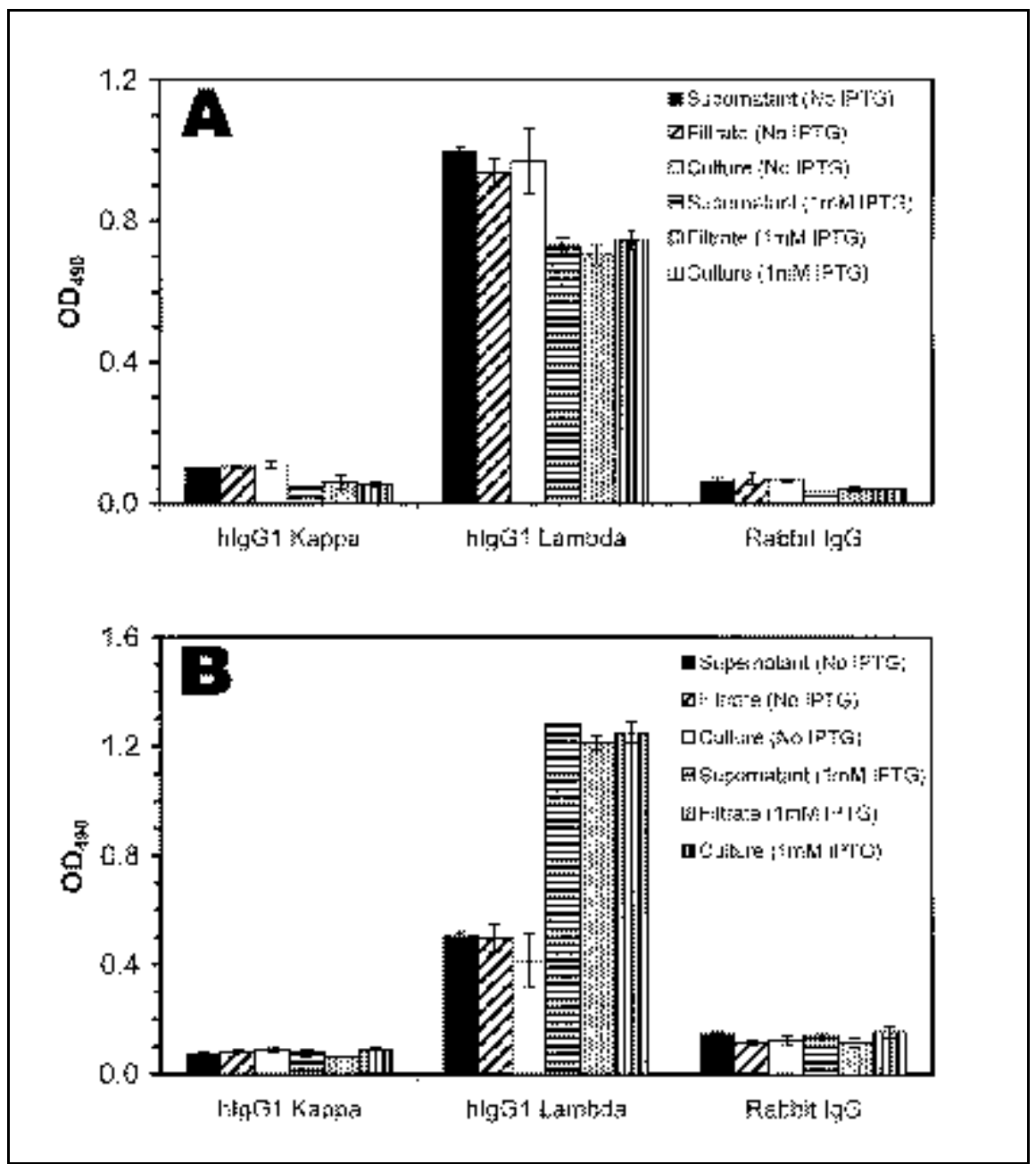

Figure 1. Binding of HP6054-phage to human lambda light chains is not affected by the presence of bacteria in the sample. Antigens were immobilized on 96-well plates (Nalge Nunc International, Roskilde, Denmark) at a concentration of $10 \mu \mathrm{g} / \mathrm{mL}$. Supernatants, clarified by centrifugation or filtration, were compared to straight bacterial cultures in a phage ELISA. Each bar represents the mean \pm SD of duplicate samples. Panel A depicts representative results from the HP6054 scFv phage, and panel B is representative of HP6054 Fab phage. 
gation. Single colonies were inoculated into $0.1-\mathrm{mL}$ cultures $(2 \times$ Yeast-Tryptone broth supplemented with $2 \%$ glucose and $100 \mu \mathrm{g} / \mathrm{mL}$ ampicillin) in a deep-well, 96-well plate and incubated at $37^{\circ} \mathrm{C}$ with shaking for 5-6 h until cultures reached mid-log phase.

Cultures then received M13KO7 helper phage (about $1 \times 10^{9}$ pfu in $5 \mu \mathrm{L}$ ) and were incubated for $1 \mathrm{~h}$ at $37^{\circ} \mathrm{C}$ with shaking. A $50-\mu \mathrm{L}$ aliquot was removed and added to a duplicate deep-well plate, and $1 \mathrm{~mL}$ media $(2 \times$ YT supplemented with $100 \mu \mathrm{g} / \mathrm{mL}$ ampicillin, 50 $\mu \mathrm{g} / \mathrm{mL}$ kanamycin with or without 1 mM IPTG) was added to the wells for overnight growth at $30^{\circ} \mathrm{C}$. Polystyrene plates were coated with protein antigens $(1-10 \mu \mathrm{g} / \mathrm{mL}$ in $0.1 \mathrm{M}$ sodium bicarbonate, $\mathrm{pH}$ 9.6) overnight at $4^{\circ} \mathrm{C}$, blocked with $3 \%$ non-fat dry milk (NFM) in TBS saline with $0.05 \%$ Tween ${ }^{\circledR} 20$, pH 7.4 (TBST), and washed three times in TBST. Aliquots of bacterial culture were removed and added to a separate microplate or to a 96-well filtration plate (MultiScreen ${ }^{\circledR}$ plates; Millipore, Bedford MA, USA). A vacuum was applied slowly, and filtrate was collected in a separate microplate.
The remainder of the culture in the deep-well plates was centrifuged at $1725 \times g$ in a centrifuge equipped with an A2MTP rotor (Eppendorf ${ }^{\circledR}$ 5804; Eppendorf Scientific, Westbury, NY, USA) for $30 \mathrm{~min}$ at room temperature. Aliquots $(80 \mu \mathrm{L})$ of clarified phage supernatant, filtrate or straight bacterial culture were added to the ELISA plate and mixed with $20 \mu \mathrm{L} 10 \% \mathrm{NFM} / 5 \times$ PBS directly in the wells. Plates were incubated with no shaking for $1.5 \mathrm{~h}$ at $37^{\circ} \mathrm{C}$, then washed four times with TBST (using a Wellwash ${ }^{\mathrm{TM}} 4$ MK2 platewasher; Labsystems, Franklin, MA, USA). HRP-conjugated anti-M13 (Amersham Pharmacia Biotech) was diluted 1:5000 into 3\% NFM/TBST and incubated in wells for $1 \mathrm{~h}$ at $37^{\circ} \mathrm{C}$. Following four washes with TBST, 100 $\mu \mathrm{L}$ of o-Phenylenediamine Dihydrochloride substrate (Sigma, St. Louis, MO, USA) was added to wells for approximately $5 \mathrm{~min}$ before stopping the reaction with $25 \mu \mathrm{L} 3 \mathrm{M} \mathrm{HCl}$. Plates were read on a microplate reader with a 490-nm filter (Bio-Rad Laboratories, Hercules, CA, USA). Assays were performed in duplicate and repeated 2-3 times with similar results.

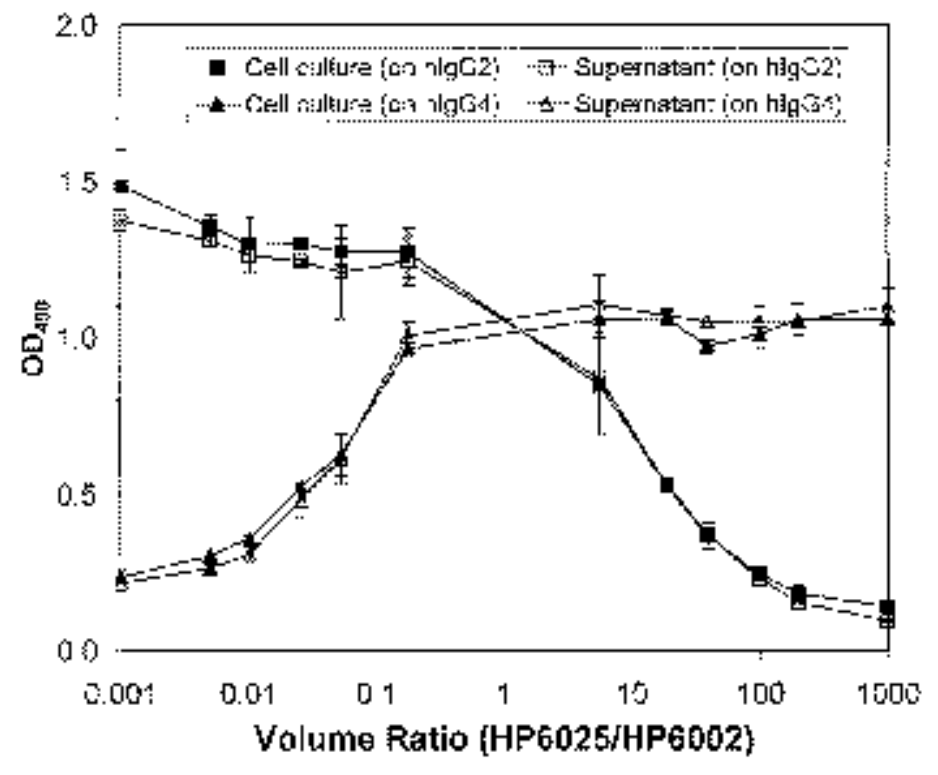

Figure 2. Sensitivity of phage ELISA is not impaired by the presence of bacteria. Overnight cultures of HP6002 scFv phage and HP6025 scFv phage were mixed at various ratios, and then supernatants or straight cultures were tested in phage ELISA against hIgG2 and hIgG4 (each present at a concentration of $10 \mu \mathrm{g} / \mathrm{mL})$. Each bar represents the mean \pm SD of duplicate samples. 
As expected, $\mathrm{scFv}$ display phage derived from HP6054 bind to the human lambda light chain antigen (in association with IgG1) but not to the kappa light chain (hIgG1 Kappa) or to rabbit IgG (Figure 1A). Filtrate generated from the same culture yielded an equivalent level of binding as observed for the supernatant. Interestingly, the unclarified culture demonstrated similar levels of binding to the immobilized antigen, which indicated that removal of bacteria by time-consuming centrifugation or costly filtration is not necessary. Also, no increase in binding to either of the two nonspecific antigens tested was observed. Similar results were observed for phage displaying a Fab also derived from HP6054 (Figure 1B). The addition of IPTG adversely affects the binding of HP6054 $\mathrm{scFv}$ phage (as a result of reduced bacterial growth and phage production; data not shown) and increases the binding of HP6054 Fab-phage (through the increased Fab:gIII fusion production; data not shown). Although the level of IPTG did affect the overall binding of the phage populations, there were no significant differences in levels of binding observed when ELISA was performed directly on bacteria-containing cultures or cultures that were clarified by centrifugation or filtration.

For some applications, assay of a polyclonal population of phage-producing bacteria would be necessary. For example, following several rounds of selection, the investigator might plan to test the population by ELISA to verify that binding members have been selected and are present in the population. To demonstrate that the bacteria remaining during the ELISA would not present a problem when low levels of binding were expected, we grew independent cultures before mixing them at various ratios. Then, we performed the ELISA on the mixed cultures or centrifugeclarified the culture supernatants derived from the same mixtures.

A mixture of cultures from two clones was used as a model system to simulate a polyclonal culture. Growth of a polyclonal culture involves competition between individuals, which affects the yield of a specific phage. However, the bias that is introduced during polyclonal growth would exist regardless of the means of analysis of that culture. $E$. coli carrying a phagemid expressing scFv derived from HP6002 (recognizing hIgG2) or HP6025 (recognizing hIgG4) were grown overnight in the absence of IPTG. Cultures that attained different densities $\left(\mathrm{OD}_{600}\right.$ for HP6002 was 1.2 and 1.0, and HP6025 was 2.1 and 3.3 in two separate trials) were mixed on the basis of volume. Final volume ratios ranged over six orders of magnitude from 0.001 to 1000 . Aliquots of the mixed culture were compared to supernatants clarified by centrifugation in the phage ELISA. Figure 2 demonstrates that the clarified phage supernatant and bacterial culture do not exhibit significant differences in binding at any of the ratios tested. Therefore, sensitivity of the ELISA does not appear to be com promised by the presence of bacteria.

Our results demonstrate the phage ELISAs can be performed directly on bacterial culture and that there is no need for clarification by centrifugation or filtration. We have successfully used cultures from scFv (five different antibodies) and Fab (two different antibodies) display phage in our ELISA (data not shown). Also, we have used this method for analysis of both peptides and proteins that are displayed on the major coat protein (gene VIII protein) of filamentous phage. To date, no significant differences between cultures and clarified supernatants have been observed for any display agents or antigens tested by this method (data not shown). This procedure has worked well with two common E. coli strains (TG1 and XL1Blue) and overnight cultures of various densities $\left(\mathrm{OD}_{600}\right.$ from 0.1 to 3.3).

While we have not tested this timesaving procedure for all available phage formats or with all displayed binding agents, we expect that it will be useful for the general screening of phage via ELISA. Importantly, elimination of centrifugation before phage ELISA will make the procedure more amenable to automation.

\section{REFERENCES}

1.de Haard, H.J., N. van Neer, A. Reurs, S.E. Hufton, R.C. Roovers, P. Henderikx, A.P. de Bruine, J.W. Arends and H.R. Hoogenboom. 1999. A large non-immunized human Fab fragment phage library that permits rapid isolation and kinetic analysis of high affinity antibodies. J. Biol. Chem. 274:18218-18230.

2.Kay, B.K., J. Winter and J. McCafferty. 1996. Phage Display of Peptides and Proteins: A Laboratory Manual. Academic Press, San Diego.

3.Reimer, C.B., D.J. Phillips, C.H. Aloisio, D.D. Moore, G.G. Galland, T.W. Wells, C.M. Black and J.S. McDougal. 1984. Evaluation of thirty-one mouse monoclonal antibodies to human IgG epitopes. Hybridoma 3:263-275.

4.Sblattero, D. and A. Bradbury. 2000. Exploiting recombination in single bacteria to make large phage antibody libraries. Nat. Biotechnol. 18:75-80.

5.Sheets, M.D., P. Amersdorfer, R. Finnern, P. Sargent, E. Lindquist, R. Schier, G. Hemingsen, C. Wong et al. 1998. Efficient construction of a large nonimmune phage antibody library: the production of high-affinity human single-chain antibodies to protein antigens (erratum appears in Proc. Natl. Acad. Sci. USA 1999 96:795). Proc. Natl. Acad. Sci. USA 95:6157-6162.

6.Smith, G.P. 1985. Filamentous fusion phage: novel expression vectors that display cloned antigens on the virion surface. Science 228:1315-1317.

Address correspondence to Dr. Paul D. Kassner, Zyomyx Inc., 3911 Trust Way, Hayward, CA 94545, USA. e-mail: pkassner@zyomyx.com

Received 20 March 2000; accepted 25 May 2000.

M. Lisa Phipps, Xiaomei Xu,
Steffen Nock and
Paul D. Kassner
Zyomyx
Hayward, CA, USA

Improved Method for the Isolation and Visualization of Terminal ProteinBound DNA Fragments in Actinomycetes

BioTechniques 29:740-745 (October 2000)

All tested wild-type Streptomyces species possess an 8-Mb linear chromosome with terminal inverted repeats (TIRs) and proteins covalently bound 